\title{
Evaluating the U.A.E.'s Orographic Cloud Seeding Techniques: A Comprehensive Analysis of Cloud Seeding Microphysics and Particle Microstructures.
}

\author{
Divraj Singh \\ Funding: The author(s) received no specific funding for this work. \\ Potential competing interests: The author(s) declared that no potential competing interests exist.
}

\begin{abstract}
Although our knowledge of cloud seeding techniques has increased substantially over the past few decades, there remains a lack of overview of its efficacy and procedures across spatial scales. (1) In this paper, we analyze the 219 cloud seeding events conducted by the U.A.E.'s National Center Of Meteorology.

(2) These events aimed to improve agriculture and mitigate the negative effects of summer drought. We analyze the microstructures of cloud particles and the microphysics of air volume and sediment interaction to identify which of the U.A.E.'s cloud seeding techniques were most efficient. First, we evaluate the conditions conducive to cloud condensation nuclei (CCN) formation through the collision-coalesce model and nucleation. Second, we consider cloud particle sedimentation, collision rates and aerosol concentrations to explain cloud behavior and cloud stability during seeding. Third, we analyze the surface tension of spherical particles and how they affect CCN grouping and collision success. Lastly, we examine current static, hygroscopic, dynamic and nanotechnology cloud seeding techniques implemented globally and identify which techniques are most suitable for the U.A.E. to adopt. We find that given the natural U.A.E. climate and the safety concerns associated with uncertain nature of emerging cloud seeding technology, it is best to use non-chemical hygroscopic methods to maximize condensation and rainfall in a sustainable way.
\end{abstract}

Key words: particles, condensation nuclei, microstructure, microphysics, rates

\section{Cloud Seeding in the U.A.E.}

Within the Middle East, U.A.E. is at the forefront of using cloud seeding technology to enhance existing water resources and to mitigate low water availability. This is primarily motivated by the fact that the U.A.E. only averages 100 millimeters of rainfall every year, most of which occurs during the winter. Launched in the 1990s, U.A.E.'s cloud seeding program began to take off in 2001 when a collaborative partnership with the U.S. National Center for Atmospheric Research (NCAR) and the U.S. Space Agency was established. In 2007, the U.A.E. set up the National Centre for Meteorology (N.C.M.) to monitor changes in the earth's atmosphere. N.C.M. currently manages all U.A.E. cloud seeding operations while overseeing a dedicated research program for rain enhancement science and offering international support/financial grants to selected researchers (4).

U.A.E. conducted 219 cloud seeding operations in the first half of 2020 during which it used 4,841 flares and 419 ground generator flares. (5) In addition, the U.A.E. currently has over 75 networked weather stations and 6 utility airplanes for cloud seeding operations. The investment impact into cloud seeding technology is evident - while in $2018,46.5 \mathrm{~mm}$ of rainfall was recorded in the U.A.E. along with 184 cloud seeding operations, this figure rose considerably in 2019 when $101.1 \mathrm{~mm}$ of rainfall occurred as a result of 247 missions. (1)

Typical cloud seeding operations have mainly focused on the mountainous regions within the north-east of the country, where warm cumulus clouds gather in the summer. Furthermore, these operations avoid using harmful chemicals and instead focus on using natural salts such as sodium and potassium chloride. Recently however, interest around new technologies involving nanoparticles has been growing. This technology consists of a titanium dioxide nanoparticle layer coated around the salt crystals. Experimental results obtained so far through laboratory testing and analysis suggest that this nanoparticle material has a much higher ability to initiate condensation of water vapor and droplet growth in the clouds than salt crystals. (3) 


\section{Cloud Formation and the Microphysics and Microstructure of Clouds}

When hot air rises up into the atmosphere, it carries water vapor up with it. As this air cools and the atmospheric pressure drops, this vapor becomes small water droplets or ice crystals that come together to form a cloud. Furthermore, vapor condenses easily into droplets when there is an abundance of solid particles to condense onto. Tiny particles such as dust and pollen are easily found in the atmosphere and are known as "condensation nuclei". Their presence allows for larger water droplets to form.

Cloud formation also requires relative humidity. Relative humidity is the partial pressure of vapor over the total pressure of air. In scientific terms, the partial pressure is the number of water molecules in a medium (air), and the total pressure is the capacity of that medium. Therefore, if the number of water molecules is high, then relative water density is high i.e. clouds will likely be generated. More specifically, cloud formation requires high relative humidity ranging from $60 \%$ to $100 \%$.

The most common way relative humidity builds up is through water vapor condensing upon particles of dust, pollen, or other condensation nuclei. Nevertheless, another way clouds form includes the collision of two large mass of air (especially warm and cold air mass) near the Earth's surface, which forces the warm air mass to rise and form a cloud. Clouds that produce rain or thunderstorms are known as nimbostratus and cumulonimbus clouds (10).

Cloud microphysics is the branch of atmospheric science concerned with the particles that make up a cloud. Relative to a cloud, these individual particles are tiny, and their sizes range from fractions of a micrometer to several centimeters. The 'microstructure' of a cloud is a specification of the concentrations, sizes, shapes, and phases of various particles and the factors that strongly influence particle behavior and cloud lifecycles. The ability of clouds to produce rain or snow, generate lightning, and alter the Earth's radiation balance stems mainly from their microstructures working in collaboration with local air motions. By characterizing the diverse microstructures of atmospheric clouds, we can understand the processes that cause them to change over time.

Cloud microphysics also involves studying the aqueous (water-like) particles that make up a cloud, their classification, and the way they change with time. These particles, either liquid or solid (ice), vary significantly in size and shape. The growth of cloud particles is determined by the physics of condensation, collision-coalescence, and nucleation. These diverse physical processes operate in a complex, dynamical environment to produce precipitation that falls to the ground.

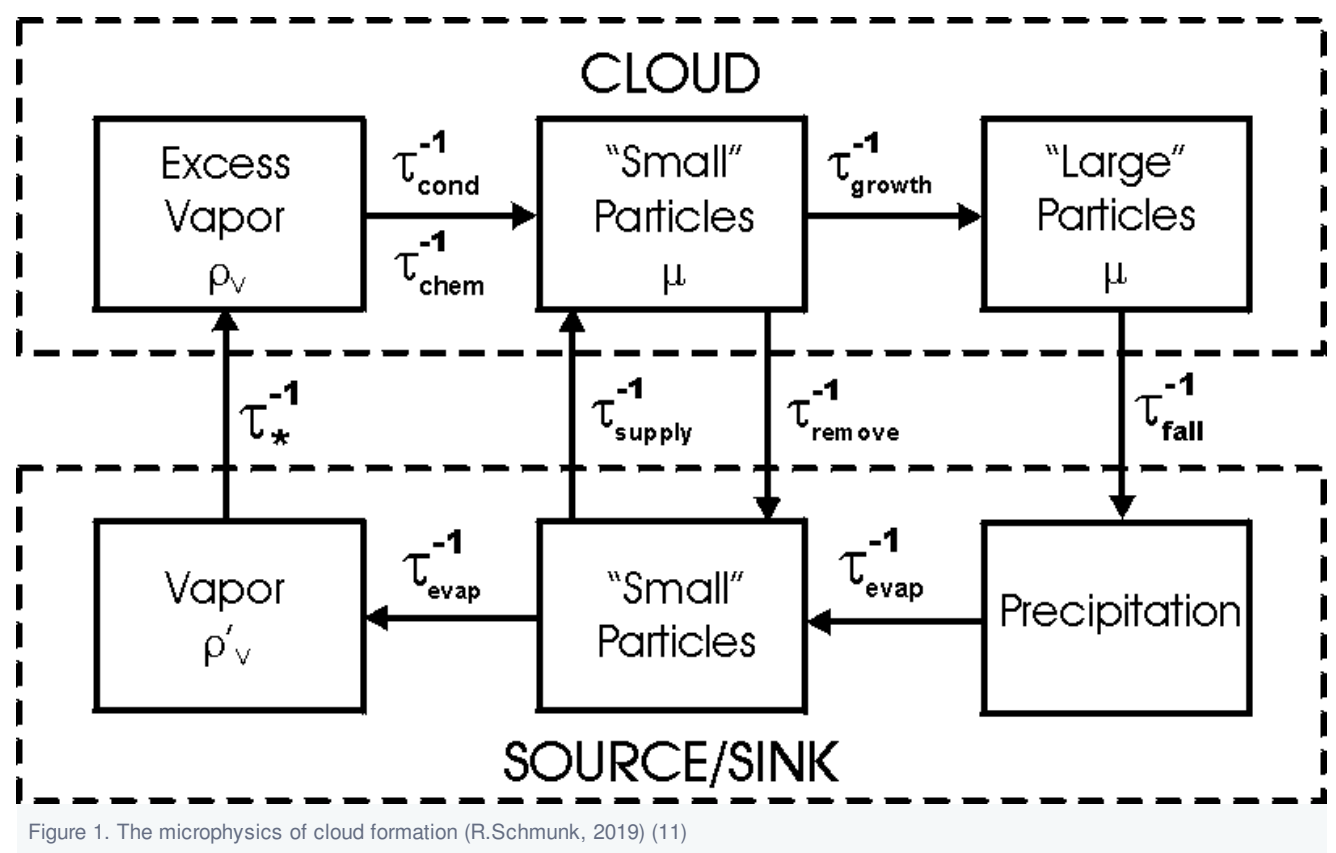


The properties of cloud particles in air can be understood in terms of the rates at which water or water mass enters and leaves the volume of air and the rate at which water mass is changed from one form to another within the volume of air (see diagram below). There are two processes by which water mass enters or leaves a particular air volume -1) atmospheric motions carrying vapor at a rate $\mathbf{r}_{*}^{-1}$ and 2) cloud particles falling fast relative to this air. If the rate of these processes is high, then excess vapor will occur.

Once excess vapor is produced, we progress to the condensation phase. This phase also has a rate $\mathrm{r}^{-1}$ cond and this rate increases with an increase of number of air particles, since the more the particles in a volume, the more likely it is that the vapor will condense around those particles. After condensation, the particles grow into droplets with a growth rate $\mathbf{r}^{-1}$ growth. If these particles are large enough then precipitation occurs and the particles fall at a rate $\mathbf{r}^{-1}$ fall. However, if these water droplets are not heavy enough, they will likely evaporate on their way down at a rate $\mathbf{r}^{-1}$ evap and repeat the cycle.

Upward air motions can reduce the sedimentation rate or even reverse its direction. The sedimentation rate (or fall rate $\mathbf{r}^{-1}$ fall) increases as particle size increases. This process is explained in Fig 1.

When the upward motions of air are more vigorous, cloud particles grow much faster. When they exceed a certain size, their falling motions relative to the air and to each other cause collisions to occur. The collision rate $\left(\mathbf{r}^{-1}\right.$ growth) of the larger particles depends on the number of particles and the speed at which they fall. If these colliding particles stick together to form an even larger particle, the rate of such collisional growth increases very rapidly. Within a cloud, there is a balance between growth by collisions (called coalescence) and removal of particles by sedimentation. Precipitation occurs when coalescence rapidly produces cloud particles that are so large their sedimentation rate exceeds their evaporation rate.

While liquid droplets stick together easily, the sticking efficiency of ice crystals depends on temperature. At very cold temperatures, ice crystals act as solid particles and do not stick well. However, at temperatures closer to the freezing point, the presence of some liquid water helps ice crystals stick together better. This is because the water gets between crystals and then freezes. Once precipitation begins to form, the cloud water is depleted and the cloud quickly dissipates. However, very violent storms produce such a large water supply and suspend precipitation-sized particles for so long, that clouds can survive for hours. (16)

As seen above, a cloud exhibits the properties of a colloidal system - a suspension of tiny particles that follow the air flow and interact with one another. Whereas the individual aqueous particles may form, grow, and subsequently disappear, the system as a whole remains microphysically stable for some time. Cloud microphysics helps us understand the specific mechanisms by which clouds form, break colloidal stability, and form precipitation. (15)

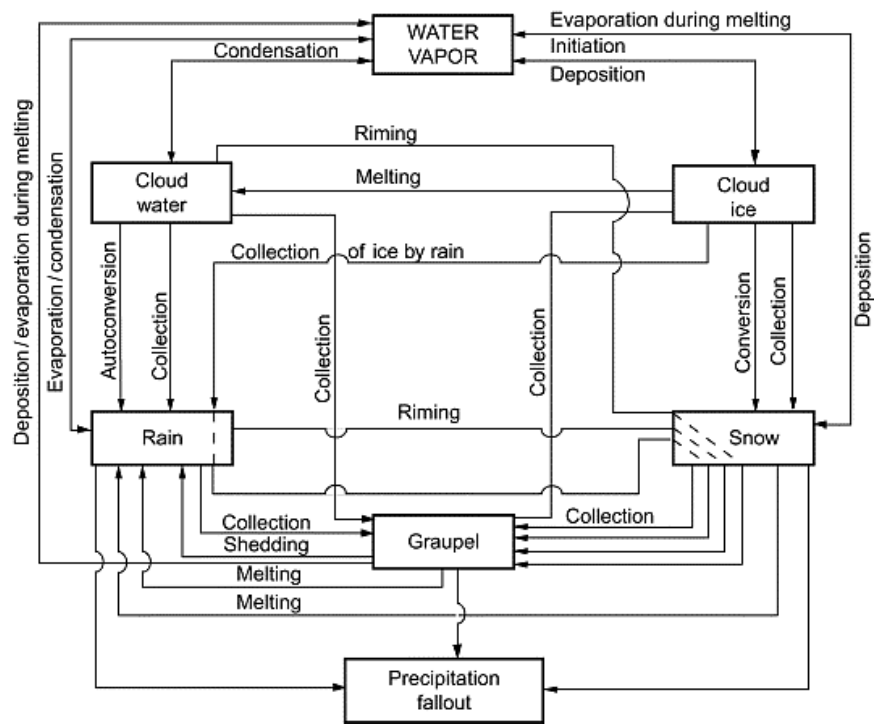


As mentioned above, the microstructure of a cloud relates to the concentrations, sizes, shapes, and phases of the various particles. The phase (solid or liquid) is the most fundamental descriptor of aqueous particles. However, non-aqueous aerosol particles are also crucial to cloud formation. Liquid condenses on soluble 'cloud condensation nuclei' (CCN), which typically contain sulfates and nitrates. Ice particles, by contrast, form on 'ice nuclei,' (insoluble aerosol particles having crystalline structures). Clouds are classified as 'warm' when most aqueous particles are liquid or 'cold,' when ice is involved (with or without liquid drops). The 'mixed-phase' of a cloud is when both liquid and solid water is present simultaneously (between the melting level $0{ }^{\circ} \mathrm{C}$ and $-40{ }^{\circ} \mathrm{C}$ i.e. the practical lower limit for liquid water to exist in the supercooled' state). (8)

\section{Droplet Formation}

The sizes and shapes of aqueous particles play important roles in cloud development. While ice particles have a wide variety of shapes, liquid drops tend to be spherical because of surface tension. Figure 3. depicts the various categories of liquid drops based on their sizes. The particle size determines its fall speed and its ability to fall against prevailing updraft speeds. Drizzle drops represent a transition between the small 'droplets' that follow air motions and the larger drops that sediment as rain. The shapes of bigger raindrops tend to be distorted due to large dynamic pressure on their lower side. (9)

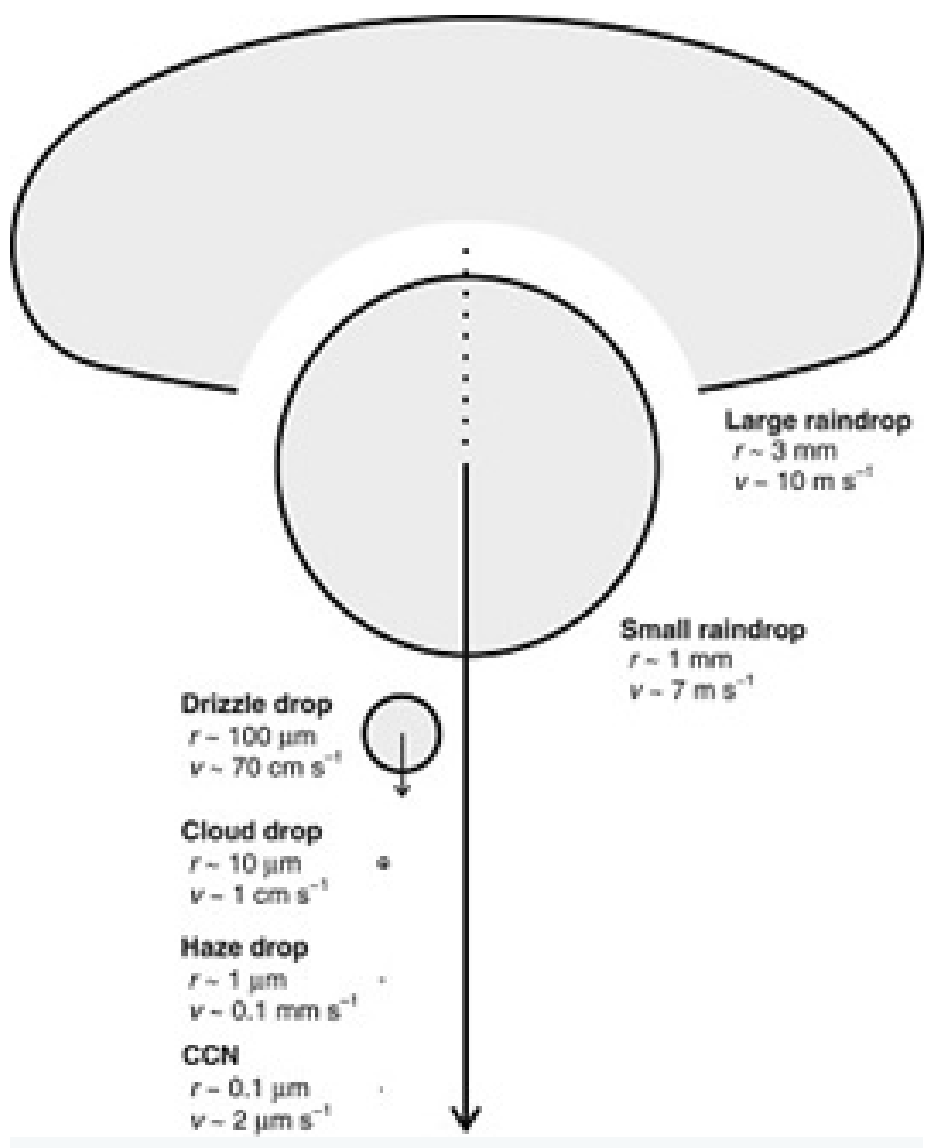

Figure 3: Droplet variations (D. Lamb et al., 2015) (9)

Various categories of liquid drops exist. Figure 3. depicts these categories with the magnitudes of drop radii $\{\mathbf{r}\}$ and fall speed $\{\mathbf{v}\}$ shown. The large raindrop is distorted due to the dynamic pressure on its underside while falling. "CCN" represents a cloud condensation nucleus, a solution droplet that serves as the initial site of condensation. (9) 
Cloud ice particles also vary enormously. These particles are initially small and monocrystalline and then subsequently grow into hexagonal prisms. In addition, a number of polycrystalline ice particles are found in cold clouds: hundreds of single crystals may clump together to form 'aggregates' (i.e., snowflakes), and supercooled cloud droplets may freeze, leading to to rimed crystals, graupel, and hail. (9)

\section{Technological Advances in Cloud Microphysics}

The role of clouds in the earth's energy balance and in climate change is the focus of intensive research. Currently, rapid technological advances are playing an increasingly large role in helping us understand the key physical processes in clouds. For example, sophisticated measurements w obtained by aircraft and radar in clouds are being used to explain the symbiotic relationship between cloud dynamics and cloud microphysics. In addition, computer models that account for the dynamics, thermodynamics, and microphysics of clouds are being used to simulate cloud lifecycles from cloud formation to collapse. Lastly, laboratory experiments in wind tunnels and cold rooms are being performed to investigate key hydrometeor growth processes.

\section{Cloud Seeding Techniques}

The study of cloud microphysics and particle formation are critical to understanding cloud seeding techniques. Rain occurs after moisture collects around cloud particles. Once clouds reaches a saturation point where it can no longer hold the moisture, rainfall occurs. Cloud seeding provides additional particles (nuclei) around which water vapor can condense to create rain. These particles can be chemicals like salt, silver iodide, potassium iodide or dry ice.

There are three methods by which cloud seeding is done and the choice of technique depends upon the type of cloud and its atmospheric condition.

\section{- Static Cloud Seeding}

This method involves spreading a chemical like silver iodide into clouds, providing crystal particles around which moisture can condense. Static cloud seeding is applied to 'cold' clouds with temperatures between $-10 \mathrm{C}$ and $-25 \mathrm{C}$. The silver iodide further increases crystal concentrations in clouds by helping to form new crystals (through particle collision) and by freezing cold droplets. Moreover, static cloud seeding does not involve air motion - all conditions are stable during the seeding process. (20)

\section{- Hygroscopic Cloud Seeding}

This method is associated with 'warm' clouds. The term hygroscopic means the tendency of a substance to absorb water moisture from air. This method increases rainfall by promoting the coalescence process whereby water particles merge during contact to form a droplet. This is achieved using hygroscopic salt nuclei either generated by a flare or spread by spraying a highly concentrated salt solution of sodium, potassium and magnesium. These flares are used to deposit salt minerals in the cloud base so that the warm air current carries them upwards into the cloud mass. Hygroscopic seeding also increases the size of the droplets (which further increases condensation. Hygroscopic cloud seeding is popular in the U.A.E. as warm clouds (convective cumulus type) are created due to its dry and hot weather. (20)

For hygroscopic cloud seeding in the U.A.E., special utility planes are fitted with flares that are loaded with salt crystals. These flares are fired into warm clouds that have an updraft. These updrafts pull the crystals into the cloud where they attract tiny particles of water making them bigger and heavier. As the heavier particles collide, they get bigger and then fall as rain after condensation and precipitation. (14) 

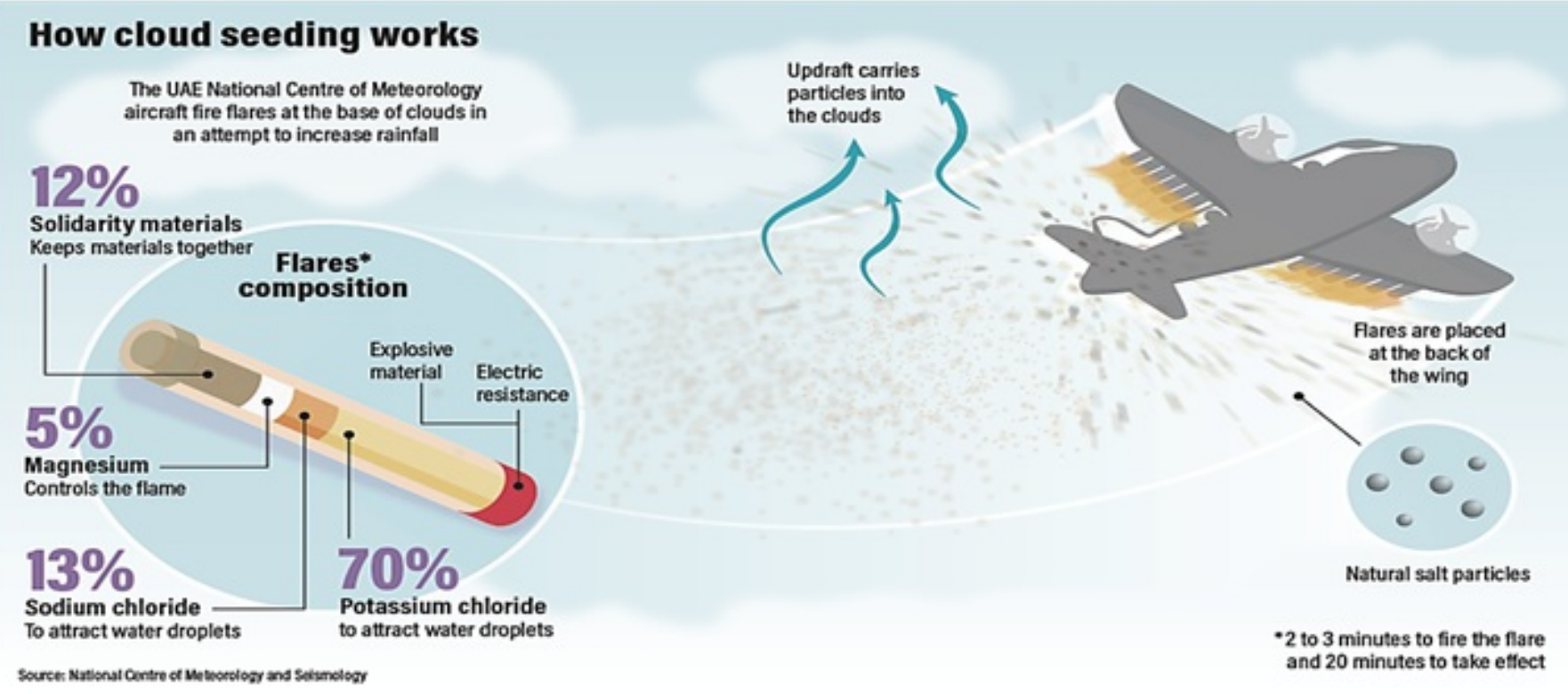

Figure 4. Hygroscopic Cloud Seeding (Gillian Duncan, 2020. the National)

Given the initial success of cloud seeding operations and the arid climate in the U.A.E., the N.C.M. has been doing several cloud seeding missions in the past 5 years, leading to a 30 to 35 per cent increase in rainfall in clean skies, and up to 15 per cent in a dusty atmosphere. According to official sources, cloud seeding in U.A.E. costs around 1 cent per cubic metre of water produced versus the 60 cents the U.A.E. pays to desalinate an equivalent amount of water. (18)

The U.A.E. has also been using ground-based seeding generators in its operations in the U.A.E. since March 2019. These ground based generators complement the traditional method of cloud seeding. The generators are fitted with 48 special flares that are loaded with salt crystals and fired into or warm clouds accompanied with updraft or rising current of air (6).

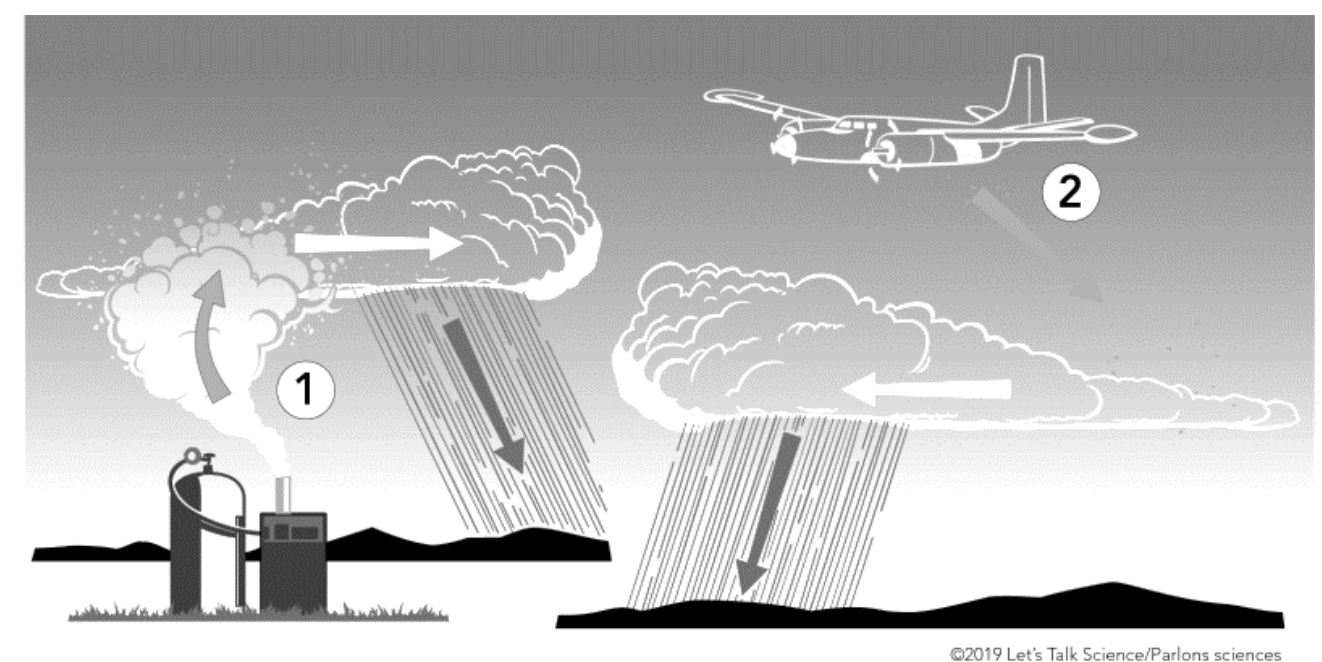




\section{- Dynamic Cloud Seeding}

This method aims to boost vertical air currents, which encourages more water to pass through the clouds. While, dynamic cloud seeding is also performed on 'cold' clouds similar to static cloud seeding, up to 100 times more ice crystals are used in dynamic cloud seeding than in the static method. The process is considered more complex and costly than static clouding seeding. (21)

\section{- Nanotechnology Cloud Seeding}

Recently, the N.C.M. has been experimenting with a new cloud seeding method involving nanotechnology. This method has been tested in the northern and eastern regions of the U.A.E. Nanotechnology involves using specially designed nanomaterials i.e. salt crystals enclosed in titanium dioxide nanoparticle veneers. These crystals are injected into the existing clouds to make rain particles denser and more likely to pour down as rainfall. (3)

\section{Conclusion and Limitations}

Cloud seeding has offered many benefits in the U.A.E. including adding to its water resources and mitigating its heavy dependence on desalination of seawater for human consumption. It has also helped the U.A.E. cope with extreme heat and dust in the air as well as helped with agriculture, rainwater harvesting and replenishing soil water levels.

Nevertheless, there exist several concerns with using cloud seeding technology, including the use of chemicals (especially silver iodide) which may cause skin rashes in people who come in contact with rain. These chemicals could also enter the water table and cause further health issues to humans, animals and plants. Other challenges include the uncertainty involved with the technology - positive results only occur under certain atmospheric conditions.

Knowledge of cloud microphysics and microstructures is crucial to mitigating some of these harmful effects. Alternatives such as spraying droplets of sea water into the atmosphere to form sea salt aerosols, or using fine dust materials including diamond dust or alumina etc. may help keep costs low while also reducing harmful health impacts. (7) Furthermore, these alternatives would also decrease unwanted side-effects like ozone depletion. (7)

\section{References}

1. The National. 2020. Cloud Seeding: It's More Complicated Than You Might Think. [online] Available at: <https://www.thenationalnews.com/U.A.E./environment/cloud-seeding-it-s-more-complicated-than-you-might-think-1.966801>

2. Ncm.ae. 2020. Ministry Of Presidential Affairs - National Center Of Meteorology - Climate Report. [online] Available at: <https://www.ncm.ae/en/climatereport.html>

3. Ncm.ae. 2020 [online] Available at: <https://www.ncm.ae/>

4. U.A.E.rep.ae. 2020. U.A.E. Research Program For Rain Enhancement Science. [online] Available at: <http://www.U.A.E.rep.ae/>

5. U.A.E.adverts.com. 2020. Gulf News U.A.E.s Dubai 2020 Latest News Today, Headlines And Stories. [online] Available at: $<$ http://www.U.A.E.adverts.com/gulf-news-U.A.E.s-dubai>

6. Carbon, D., 2020. The Sustainabilist. [online] Issuu. Available at: <https://issuu.com/dccepublications/docs/sme_issue1_0.9_ra>

7. Conca, J., 2020. Seeding The Clouds - Should We Mess With Our Earth's Climate?. [online] Forbes. Available at: 
<https://www.forbes.com/sites/jamesconca/2017/06/22/seeding-the-clouds-should-we-mess-with-our-earths-climate/>

8. Sciencedirect.com. 2020. International Geophysics / Cloud Dynamics / Sciencedirect.Com By Elsevier. [online] Available at: <https://www.sciencedirect.com/bookseries/international-geophysics/vol/104>

9. Sciencedirect.com. 2020. Cloud Microphysics - An Overview / Sciencedirect Topics. [online] Available at: <https:/www.sciencedirect.com/topics/earth-andplanetary-sciences/cloud-microphysics>

10. Scied.ucar.edu. 2020. UCAR Center For Science Education /. [online] Available at: <https://scied.ucar.edu/>

11. Isccp.giss.nasa.gov. 2020. ISCCP:Cloud Microphysics - Part 1. [online] Available at: <https://isccp.giss.nasa.gov/analysis/climanal8.html> [Accessed 9 November 2020].

12. Climate.ncsu.edu. 2020. Cloud Seeding / North Carolina Climate Office. [online] Available at: <https://climate.ncsu.edu/edu/CloudSeeding>

13. Let's Talk Science. 2020. What Is Cloud Seeding?. [online] Available at: <https://letstalkscience.ca/educational-resources/stem-in-context/what-cloudseeding>

14. Duncan, T.G, 2020. How Does Cloud-Seeding In The U.A.E. Work?. [online] The National. Available at: <https://www.thenationalnews.com/U.A.E./environment/how-does-cloud-seeding-in-the-U.A.E.-work-1.811961>

15. Fan, J., Leung, L., Rosenfeld, D., Chen, Q., Li, Z., Zhang, J. and Yan, H., 2020. Microphysical Effects Determine Macrophysical Response For Aerosol Impacts On Deep Convective Clouds.

16. Schmunk, R., 2020. ISCCP:Cloud Microphysics - Part 1. [online] Isccp.giss.nasa.gov. Available at: <https:/isccp.giss.nasa.gov/analysis/climanal8.html>

17. Gulftoday.ae. 2020. NCM Carries Out 95 Cloud Seeding Operations In Q1 Of 2020. [online] Available at: <https://www.gulftoday.ae/news/2020/04/12/nmccarries-out-95-cloud-seeding-operations-in-q1-2020>

18. A blog about homes, trends, tips \& life in the U.A.E. | MyBayut. 2020. All About Cloud Seeding In U.A.E.: Methods, Importance \& More - Mybayut. [online] Available at: <https://www.bayut.com/mybayut/all-about-cloud-seeding-U.A.E./>

19. The National. 2020. U.A.E. To Take Cloud Seeding Research To New Heights. [online] Available at: <https://www.thenationalnews.com/U.A.E./environment/U.A.E.-to-take-cloud-seeding-research-to-new-heights-1.967496>

20. Malik, S., 2020. [online] Available at: <https://www.researchgate.net/publication/328718655_Cloud_Seeding_Its_Prospects_and_Concerns_in_the_Modern_World-A_Review>

21. 2020. [online] Available at: <https://www.researchgate.net/publication/249614986_A_Review_of_Cloud_Seeding_Experiments_to_Enhance_Precipitation_and_Some_New_Prospects> 